\title{
Locating the source field lines of Jovian decametric radio emissions
}

\author{
YuMing Wang ${ }^{1,2,3^{*}}$, XianZhe Jia ${ }^{4}$, ChuanBing Wang ${ }^{1,2}$, Shui Wang ${ }^{1,2}$, and Vratislav Krupar, ${ }^{5,6,7}$ \\ ${ }^{1}$ Chinese Academy of Sciences Key Laboratory of Geospace Environment, School of Earth and Space Sciences, University of Science and Technology of China, \\ Hefei 230026, China; \\ ${ }^{2}$ Chinese Academy of Sciences Center for Excellence in Comparative Planetology, University of Science and Technology of China, Hefei 230026, China; \\ ${ }^{3}$ Mengcheng National Geophysical Observatory, School of Earth and Space Sciences, University of Science and Technology of China, Hefei 230026, China; \\ ${ }^{4}$ Department of Climate and Space Sciences and Engineering, University of Michigan, Ann Arbor, MI 48109-2143, USA; \\ 5Universities Space Research Association, Columbia, Maryland, USA; \\ ${ }^{6}$ NASA Goddard Space Flight Center, Greenbelt, Maryland, USA; \\ ${ }^{7}$ Department of Space Physics, Institute of Atmospheric Physics, The Czech Academy of Sciences, Prague, Czech Republic
}

\section{Key Points:}

- A method is established to locate the source regions of Jovian DAM emissions based on radio observations

- Multiple spacecraft from different perspectives can be used to infer the evolution of the DAM source

- For the event studied in this paper, the footprints of the active field lines are about 32 degrees ahead of lo's footprints in longitude

Citation: Wang, Y. M., Jia, X. Z., Wang, C. B., Wang, S., and Krupar, V. (2020). Locating the source field lines of Jovian decametric radio emissions. Earth Planet. Phys., 4(2), 95-104. http://doi.org/10.26464/epp2020015

\begin{abstract}
Decametric (DAM) radio emissions are one of the main windows through which one can reveal and understand the Jovian magnetospheric dynamics and its interaction with the moons. DAMs are generated by energetic electrons through cyclotron-maser instability. For lo (the most active moon) related DAMs, the energetic electrons are sourced from lo volcanic activities, and quickly trapped by neighboring Jovian magnetic field. To properly interpret the physical processes behind DAMs, it is important to precisely locate the source field lines from which DAMs are emitted. Following the work by Hess et al. (2008, 2010), we develop a method to locate the source region as well as the associated field lines for any given DAM emission recorded in a radio dynamic spectrum by, e.g.,

Wind/WAVES or STEREO/WAVES. The field lines are calculated by the state-of-art analytical model, called JRM09 (Connerney et al., 2018 ). By using this method, we may also derive the emission cone angle and the energy of associated electrons. If multiple radio instruments at different perspectives observe the same DAM event, the evolution of its source region and associated field lines is able to be revealed. We apply the method to an lo-DAM event, and find that the method is valid and reliable. Some physical processes behind the DAM event are also discussed.
\end{abstract}

Keywords: radio decametric emissions; Jovian magnetosphere; energetic electrons

\section{Introduction}

Jupiter is the largest planet in our solar system with fastest rotation and strongest magnetic field. Its surface magnetic field is as strong as tens of Gauss, which leads to the most intense planetary decametric (DAM) radio emissions (e.g., Zarka, 1998, and references therein). Like the auroral kilometric radiations (AKRs) at the Earth, Jovian DAM emissions are caused by high energetic electrons through Cyclotron-Maser (CM) instability (e.g., Wu and Lee, 1979; Lecacheux, 1988; Dulk et al., 1994; Queinnec and Zarka, 1998; Treumann, 2006; Hess et al., 2008). The energy of electrons emitting DAMs is believed to be generally above $0.5 \mathrm{keV}$ (Waite et

Correspondence to: Y. M. Wang, ymwang@ustc.edu.cn

Received 19 AUG 2019; Accepted 05 FEB 2020.

Accepted article online 28 FEB 2020.

(C)2020 by Earth and Planetary Physics. al., 1988; Zarka et al., 1996; Hess et al., 2008). Since Jupiter's magnetosphere holds cold and strongly magnetized plasmas with the electron plasma frequency much smaller than electron cyclotron frequency (i.e., $\left.f_{\mathrm{pe}} / f_{\mathrm{ce}}<0.1-0.2\right)$ (e.g., Connerney, 1992; Bagenal, 1994), Jovian DAMs beam along the narrow walls (e.g., Kaiser et al., 2000; Panchenko and Rucker, 2016) of a widely-opened hollow cone according to the basic properties of CM emissions (e.g., Queinnec and Zarka, 1998; Hess et al., 2008; Hess et al., 2014; Lamy et al., 2008; Lamy et al., 2013). This process leaves DAM emissions an 'arc' shaped drift pattern in the radio dynamic spectrum, ranging roughly from one to several tens of $\mathrm{MHz}$ depending on the magnitude of the magnetic field and the energy of the associated electrons.

On the other hand, unlike AKRs, which are mainly driven by the external forcing, i.e., the solar wind, the Jovian DAMs, especially 
the lo-related DAMs occurring in the inner magnetosphere, are mostly driven by internal processes due to the strong magnetic field (e.g., Hill et al., 1983; Zarka, 1998; Cowley and Bunce, 2001; Kivelson and Southwood, 2005). lo, with more than 400 volcanoes, is the most active moon of Jupiter, supplying large amount of plasmas into magnetosphere (Schneider and Bagenal, 2007). These loaded plasmas are picked up by and corotate with the Jovian magnetosphere, and drive many dynamic processes there including DAMs. Thus, DAM emissions are of great interest as they carry important information of the Jovian magnetospheric dynamics, and can be detected by remote radio instruments on ground and/or on spacecraft.

One of the key information of DAMs is the source location as well as the associated field lines from which they are emitted. According to the 'arc'-shaped drift pattern of DAMs on radio dynamic spectra, we can know if they come from the vicinity of west limb (look like 'a front bracket') or east limb ('a rear bracket') of Jupiter. However, such a determination is too rough to reveal detailed processes behind the observed features. For example, the plasma in the flux tube linked to lo is believed to be the source of loDAMs. However, the strongest emission of lo-DAMs typically shifts toward smaller central meridian longitudes (CML) in System III, i.e., moving ahead of lo in the direction of lo orbiting around Jupiter (e.g., Zarka, 1998; Imai et al., 2002; Hess et al., 2010). Such a shift, or lead angle between the active field line and lo flux tube, can be explained by the presence of Alfvén wings arising from lo's interaction with the sub-Alfvénic flow of the Jovian corotating plasma (e.g., Hill et al., 1983; Jacobsen et al., 2007; Bonfond et al., 2008; Bonfond et al., 2009).

To locate the DAM radio source, Imai et al. $(1997,2002)$ developed a model based on the observed modulation lanes in the high frequency range of DAM spectra (e.g., Riihimaa, 1968; Riihimaa, 1978). In that model, the source is assumed to be a linear and thin structure along Jupiter's tilted dipole magnetic field that corotates with lo. The emission cone angle and the associated longitude of the emission can be derived from this model. Later, Hess et al. $(2008,2010)$ established a relation between the velocity of energetic electrons and the emission cone angle based on the lose-cone distribution, and then developed a general simulation code called 'ExPRES' (Exoplanetary and Planetary Radio Emission Simulator). In this method, the lead angle between the active field line and lo in longitude and the energy of the associated electrons are two free parameters obtained by fitting observed DAM arcs. Here, inspired by Hess et al. $(2008,2010)$, we propose a similar method to determine the source region of a DAM, in which a state-of-art Jovian magnetic field model (Connerney et al., 2018) is used. As will be shown below, the core function is the same as that of Hess et al. $(2008,2010)$, and the input is also just the observed DAM arcs in a broad frequency band, but the procedure is different and therefore results in similar but not exactly the same output. Our method, for example, does not introduce the lead angle between the active field lines and lo as a free parameter when locating DAM source, though we may derive it after all the source field lines have been identified. With observations from multiple spacecraft at different perspectives, the evolution of the DAM source, including, e.g., the apparent rotation speed of the source, the range of the active field lines, and the energy of asso- ciated electrons, can be revealed. The details of the method are presented in the next section. In Sections 3 and 4, we will verify the method by applying it to an lo-DAM and show some interesting processes behind the lo-DAM. A summary and discussion is then given in Section 5 .

\section{Method}

The basic idea of our locating method is that a DAM emission is not isotropic and the frequency depends on the local magnetic field strength owing to the fact that DAMs are fundamental $X$ mode through the CM instability (e.g., Lecacheux, 1988; Zarka, 1998). From a radio dynamic spectrum, we can read the frequency-time drift pattern of a DAM as shown in Figure 1. First, we may immediately know that the DAM comes from eastern/western hemisphere if the drift pattern looks like a rear/front bracket (e.g., Hess et al., 2014). Second, the recorded frequency can be used to derive the magnetic field strength in the source region where the DAM is emitted according to the electron cyclotron frequency formula

$$
B=0.358 f
$$

in which $B$ is in units of Gauss and $f$ in units of $\mathrm{MHz}$. It should be noted that the observed frequency $f$ is larger than but very close to the local electron cyclotron frequency $f_{\text {ce }}$ based on theory, the magnetic field $B$ is therefore slightly overestimated, which is ignored in our method. After converting the observed frequency to magnetic field strength, we may search Jupiter's eastern or western magnetosphere for all the places matching the requirement of $B$.

Jupiter's magnetic field is calculated by using the newest model based on the observations from Juno's first nine orbits (Bolton et al., 2017). The model consists of two components. One is the potential field originating from the interior of Jupiter, of which the spherical harmonic coefficients extending to the order of 10 were updated by Connerney et al. (2018), and referred as 'JRM09' model field (Juno Reference Model through Perijove 9). The other is the external field due to the presence of the magnetodisc (or the current sheet) from 5 to several tens of Jovian radii (Connerney et al., 1981). Here we use the typical values of the parameters of the magnetodisc model, i.e., the total current related coefficient $\mu_{0} I_{0} / 2=225$, the inner radius of the disc $r_{a}=5 R_{\mathrm{J}}$, the outer radius $r_{b}=50 R_{\mathrm{J}}$, and semi-thickness $D=2.5 R_{\mathrm{J}}$. A more accurate formula to calculate the external field was given by Giampieri and Dougherty (2004), and is adopted here. By using the JRM09 plus magnetodisc model, we trace magnetic field lines from the $1 / 15.4$ flattened surface of one Jovian radius $\left(R_{\mathrm{J}}\right)$ to get the $\boldsymbol{B}$ vectors including the strength and direction in the Jovian magnetosphere. The spatial step to trace the field lines is ranged between 0.01 and $0.5 R$, depending on the curvature of the local field line. To avoid possible biases from a non-uniform distribution of footpoints of the field lines, we use a geodesic polyhedron (https://en.wikipedia. org/wiki/Geodesic_polyhedron) with 40962 vertices (see Figure 2 ), from which we trace all the field lines. The number of footpoints makes the angle between neighboring points be about $1^{\circ}$, which meets the angular resolution required from the thickness of the wall of the emission cone as discussed below. 




Figure 1. From top to bottom, the panels show the radio dynamic spectra from Wind/WAVES, ST-A/WAVES and ST-B/WAVES, respectively.

A DAM is emitted along the narrow wall of a hollow cone, meaning that an observer can only receive the emission from a certain direction. The emission cone angle, $a$, generally varies between $60^{\circ}-90^{\circ}$ (e.g., Queinnec and Zarka, 1998; Hess et al., 2008; Hess et al., 2014; Lamy et al., 2008; Lamy et al., 2013) with the wall thickness less than $2^{\circ}$ (e.g., Kaiser et al., 2000; Panchenko and Rucker, 2016). This is another constraint for our method to locate the DAM source. For each candidate point selected based on the magnetic field strength, we know the direction of the point-observer line, and therefore the angle, $a_{p}$, between the line and the local field line where the point locates. These candidate points are then further filtered by checking if the associated $a_{p}$ falls in the range of expected $a$ with the thickness of $2^{\circ}$.

The range of the emission angle $a$ is too wide to make precise filtering. Thus, we follow Hess et al. $(2008,2010)$ using the formula below to constrain the value of $a$

$$
a=\arccos \left[\frac{v}{c}\left(1-\frac{f_{\mathrm{ce}}}{f_{\mathrm{ce}, \mathrm{max}}}\right)^{-1 / 2}\right],
$$

in which $v$ is the speed of the energetic electrons, $c$ is the light speed and $f_{\mathrm{ce}, \max }$ is the maximum of the electron cyclotron frequency that the electrons can reach along the active field line, i.e., the high cut-off, typically choosing the value at the top of ionosphere. For each field line on which there are some candidate points, we know the values of $a_{p}$ of all these candidate points and the associated magnetic field strength, i.e., the frequency $f$, and can fit the data pairs of $a_{p}$ and $f$ with Equation (2) by assuming that the energy, i.e., $v / c$, of the electrons generating the DAM emission remain unchanged when moving along this field line. Based on the previous studies mentioned before, our procedure limits the range of $a$ to $50^{\circ}-90^{\circ}$ and the energy of electrons to above $0.2 \mathrm{keV}$ (or the speed $v>0.05 \mathrm{c}$ ). If the fitting can reach a converged value of $v / c$, say its standard deviation is less than 0.01 in our procedure, the candidate points as well as the associated field line are finally selected.

It should be noted that, for one DAM arc in the radio dynamic spectrum, the method may find a set of field lines matching the above criteria: (1) the strength of the field line covers the corresponding frequency swept by the DAM arc, and (2) the emission cone angles and corresponding frequencies of all the candidate 


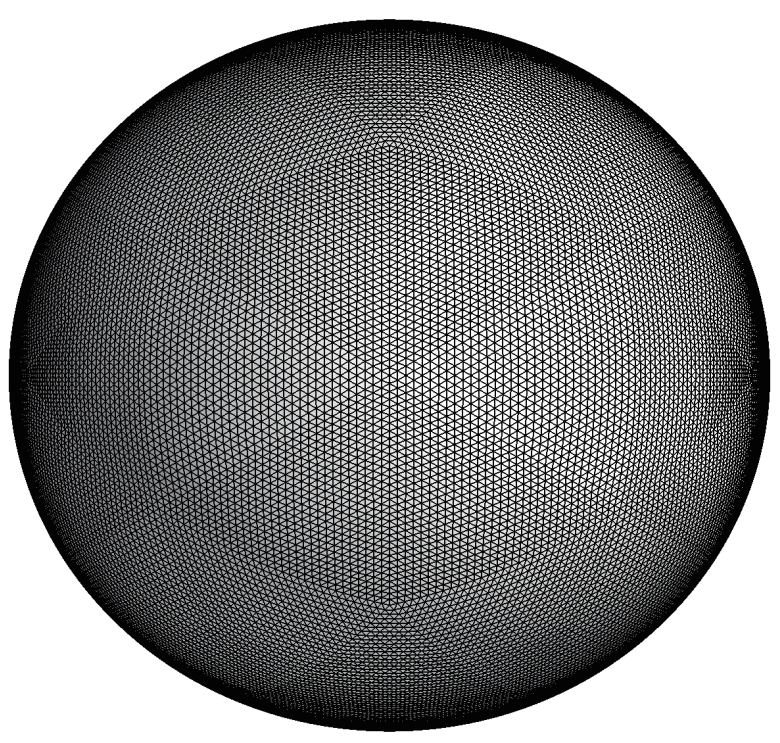

Figure 2. The flattened geodesic polyhedron with 40962 vertices (see main text for details). Since Jupiter is an ellipsoid with the equatorial radius of $1 R_{\mathrm{J}}=71492 \mathrm{~km}$ and polar radius of $0.935 R_{\mathrm{J}}$, the polyhedron is flattened by a factor of $1 / 15.4$.

points on each field line can be well fitted by Equation (2). On the selected field lines, the obtained electron velocity may be different. But we think that the electrons on these identified field lines could all contribute to the observed DAM emission, and therefore could be all real sources.

The above procedure is based on the observed radio dynamic spectrum from one spacecraft. Through it, we may locate the source region of a DAM emission with the following parameters derived: (1) the position of the source in the three-dimensional space, (2) the information of the associated field lines including the longitudes and latitudes of their footprints, (3) the emission cone angle and (4) the energy or speed of the associated electrons. If there are multiple spacecraft at different perspectives, e.g., Wind, STEREO-A and B (ST-A and ST-B for short), we may further obtain the changes of these derived parameters, reflecting the evolution of the emission source.

Besides, one more constraint is the polarization of emission. As DAMs are the result of CM instability, the right-handed polarized DAMs originate from the northern hemisphere and the lefthanded polarized DAMs from the southern hemisphere (e.g., Zarka, 1998; Treumann, 2006). However, not all of the radio observations have the information of polarization. For those having no polarization measurements, we either try both hemispheres or pre-determine the hemisphere based on other information, e.g., the tilt angle of the magnetic pole respective to the observer. If the north pole tilts toward the observer, we tend to believe that the emission comes from the northern hemisphere.

\section{An Io-DAM on 2014 March 14}

To verify the method, we apply it to an lo-DAM event. For an loDAM, we know the position of lo and therefore roughly know where its related DAM should be emitted. The lo-DAM observed by Wind/WAVES (Bougeret et al., 1995) and ST-A and B/WAVES (Bougeret et al., 2008) on 2014 March 14 (see Figure 1) is selected for the test. In the radio dynamic spectra, we can see clearly an intense radio signature drifting from about 5 to $16 \mathrm{MHz}$ within about 25 minutes. The Wind/WAVES first received the DAM emission at about 17:22 UT with a long-lasting radio noisy burst appearing below $5 \mathrm{MHz}$. The ST-A/WAVES received the DAM emission about two hours later at about 19:24 UT. The ST-B/WAVES received the signal at about 19:44 UT. The time shifts of the DAM signature on the three spectra are due to the configuration of the three spacecraft relative to Jupiter as shown in Figure 3. The emission cone of the DAM first swept the Earth, and then ST-A and ST$B$. The time difference due to the different travel distances of the light from Jupiter to the Earth, ST-A and B is several minutes, which is much shorter compared to the observed time shifts among the observers. After correcting for the difference due to the light travel time, we deduce that the source of DAM rotated at a speed much slower than Jupiter itself, but was very close to the rotation speed of lo. Thus, it is lo-related.



Figure 3. Positions of the Earth, ST-A, ST-B and Jupiter in the heliocentric inertial coordinate $(\mathrm{HCl})$ system. The big yellow dot indicates the Sun.

A more accurate estimation of the rotation speed of the DAM source is made by calculating the two-dimensional (2D) cross-correlation coefficient $(c c)$ between the radio dynamic spectra from two spacecraft, say Wind and ST-B. We set a 21-minute wide and 5 $\mathrm{MHz}$ high box locating between 5 and $10 \mathrm{MHz}$, where the DAM signature is the most significant in both spectra (see Figure 1), and run the box through both radio dynamic spectra with a certain time shift (or the time lag of the box in one spectrum compared to the other) to calculate the $c c$ value. The width of the box is set to be 21 minutes because it can roughly cover the track of the DAM 
in the spectra. For a given time lag, we may get a time sequence of the $c c$ values. Then we adjust the time lag to obtain a 2D cc distribution as shown in Figure 4, in which the horizontal axis is the time corresponding to the time recorded by Wind/WAVES and the vertical axis on the left gives the time lag. A negative value of the time lag means that the running box in the ST-B's spectrum lags behind that in the Wind's spectrum.



Figure 4. A $2 D$ distribution of the correlation coefficients made between the spectra from Wind/WAVES and ST-B/WAVES. The horizontal axis gives the time at Wind, and the vertical axis on the left indicates the time lag of ST-B. For each time lag, we calculate the corresponding rotation speed in units of self-rotational angular speed of Jupiter, $\Omega_{\mathrm{J}}$, scaled by the vertical axis on the right. The cyan line denotes the rotation speed of lo. See main text for more details.

One can find one and only one significantly high cc region around the time when the DAM appeared in the Wind's spectrum. The time lag of the high cc region is about -142 minutes, including the time difference of about 5 minutes due to the light travel. Since the angular separation of the Wind and ST-B with respective to Jupiter is about $21.5^{\circ}$, we may derive that the rotation speed of the DAM source, $\Omega_{c C}$ is about $0.255 \Omega_{\mathrm{J}}$, in which $\Omega_{\mathrm{J}} \approx 0.6^{\circ} / \mathrm{min}$ is the self-rotational angular speed of Jupiter, as scaled by the vertical axis on the right-hand side. The rotation speed of Io, $\Omega_{\text {lo }}$, is about $0.23 \Omega$, which is marked by the cyan dashed line. According to the statistical study of UV observations of Jupiter from Hubble Space Telescope (Grodent et al., 2008; Bonfond et al., 2009), the rotation speed of lo's footprint (IFP) magnetically mapping on the northern ionosphere, $\Omega_{\mathrm{IFP}}$, is about $0.55 \Omega_{\mathrm{J}}$ during the event. The estimated rotation speed of the DAM source is bounded between the two speeds, suggesting that the DAM emission was lo related.

We then apply our method to this event to identify the source region from which the DAM was emitted. From the drift shape of this DAM emission, we know it came from the western hemisphere. We do not have the polarization measurements of the event, but on 2014 March 14 the northern magnetic pole was tilted toward the spacecraft. Thus, we believe that the DAM emission should come from the northern hemisphere. Previous studies also suggested that DAMs are more likely to come from the northern hemisphere under such configurations (e.g., Carr et al., 1983). We use the drifting tracks above $5 \mathrm{MHz}$ for this event, as they are most clear in the radio dynamic spectra. These tracks have a width of about 10 minutes at a given frequency and span over about 25 minutes from 5 to $16 \mathrm{MHz}$. The value of $f_{\mathrm{ce}, \max }$ is set to be the value at the footprint of each field line on the $1 / 15.4$ flattened surface of one $R_{\mathrm{J}}$, as an approximation of the frequency at the top of ionosphere. Then we input all this information into our method, and search all the field lines starting from the vertices (see Figure 2) on the western and northern hemisphere.

The located DAM source corresponding to the frequency range of 5-16 MHz is shown in Figure 5. From the upper to lower panels, there are the results based on the radio dynamic spectra from the Wind, ST-A and ST-B spacecraft. It is found that the radio source at a certain time is successfully confined in a relatively small region as indicated by the blue-red colors. The red symbols correspond to the emissions at $16 \mathrm{MHz}$. Their heights from the one- $R$, surface were about $0.1 R$, when Wind/WAVES received the signals and increased to about $0.25 R$, when ST-A and B/WAVES received the signals. The blue symbols correspond to the emissions at $5 \mathrm{MHz}$ with the height of about $0.65-0.75 R$, during the event. All of these heights are well above Jupiter's ionosphere. Besides, the source field lines concentrate in a narrow angle in longitude, forming a fan-shaped sector. This sector was moving ahead of lo as expected. A more detailed analysis is given below.

\section{Analysis of the lo-DAM Source}

The footprint of the identified radio source on the 1/15.4 flattened surface at one $R_{\mathrm{J}}$ is displayed in Figure 6. Our method suggests that the source field lines were located near the edge of the region that contains the strongest magnetic field in the northern hemisphere during 16:42-17:15 UT (the left panel). Note that all the times hereafter are the times at Jupiter if not specified. About two hours later, the footprint moved toward the strongest magnetic field region. The change of the longitudes of these identified source field lines is shown clearer in the right panel, decreasing from about $248^{\circ}$ at Wind to about $210^{\circ}$ at ST-B. Compared with the IFPs obtained from UV observations (Bonfond et al., 2009), we may find that the footprints of the source field lines located ahead of the IFPs (indicated by the orange line segments in the left panel of Figure 6) with a nearly constant lead angle of about $32^{\circ}$ in longitude. It should be noted that the derived longitude only shows changes during the first 10 minutes and the last 10 minutes. This is because the DAM pattern on the radio dynamic spectra is an about 10-minute-width drifting feature over 35 minutes; during the middle 15 minutes, the inputs of our method are the same and therefore the derived parameters are the same.

The apparent rotation speed of the footprint relative to Jupiter's magnetic field was about $38^{\circ} / 137 \mathrm{~min} \approx 0.28^{\circ} / \mathrm{min}$, or converting to the rotation speed in the inertial coordinates, $\Omega_{\mathrm{fp}} \approx 0.55 \Omega_{\mathrm{J}}$, the same as the apparent longitudinal speed $\Omega_{\text {IFP }}$ on the northern hemisphere estimated in the last section. This value is larger than $\Omega_{c c} \approx 0.255 \Omega \mathrm{J}$ derived from $2 \mathrm{D}$ correlation analysis above. The reason is that Jupiter's magnetic field is not a pure dipole field with its axis aligned with Jupiter's rotational axis and the emission cone angles changed. To explain $\Omega_{c c}<\Omega_{\mathrm{fp}}$, the angle of main emissions should become smaller with time. As can be seen in Figure 8 , the average values of the derived emission angles did change. For instance, in the left panel, the average and maximum values of the emission angle both decreased by about $2^{\circ}$ during the 137 minutes.

The longitudes of the tops of these source field lines are shown in the left panel of Figure 7. It is found that the longitudes of tops are 


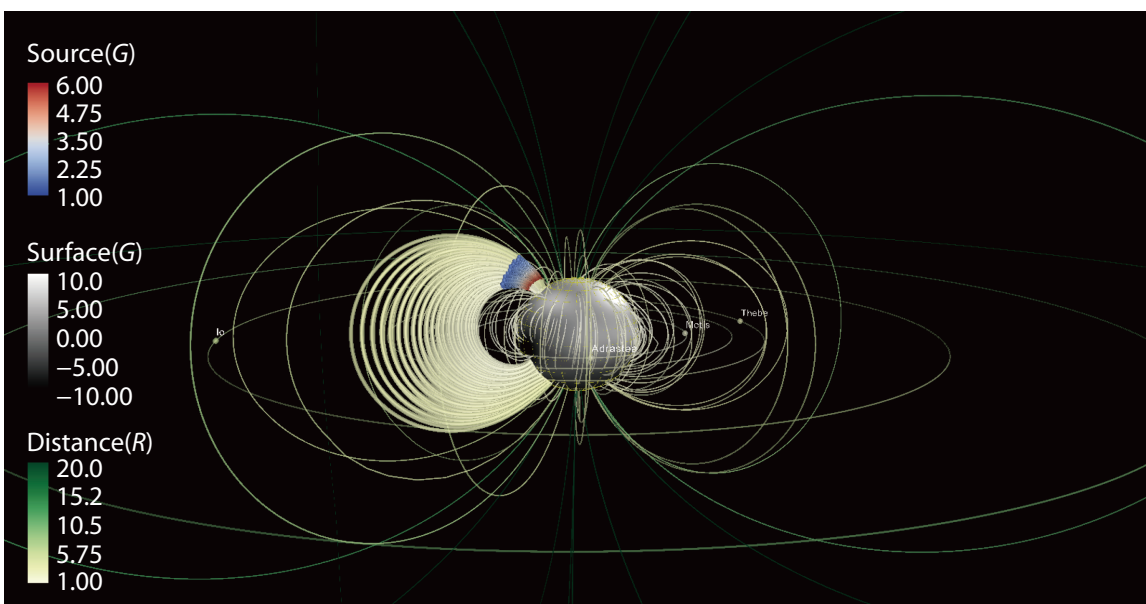

2014-03-14 17:06:35.000, longitude of 331.5



1.00

Surface $(G)$

10.0

5.00

0.00

$-5.00$

$-10.00$

Distance $(R)$

20.0

15.2

10.5

5.75
1.00

2014-03-14 18:56:35.000, longitude of 281.0

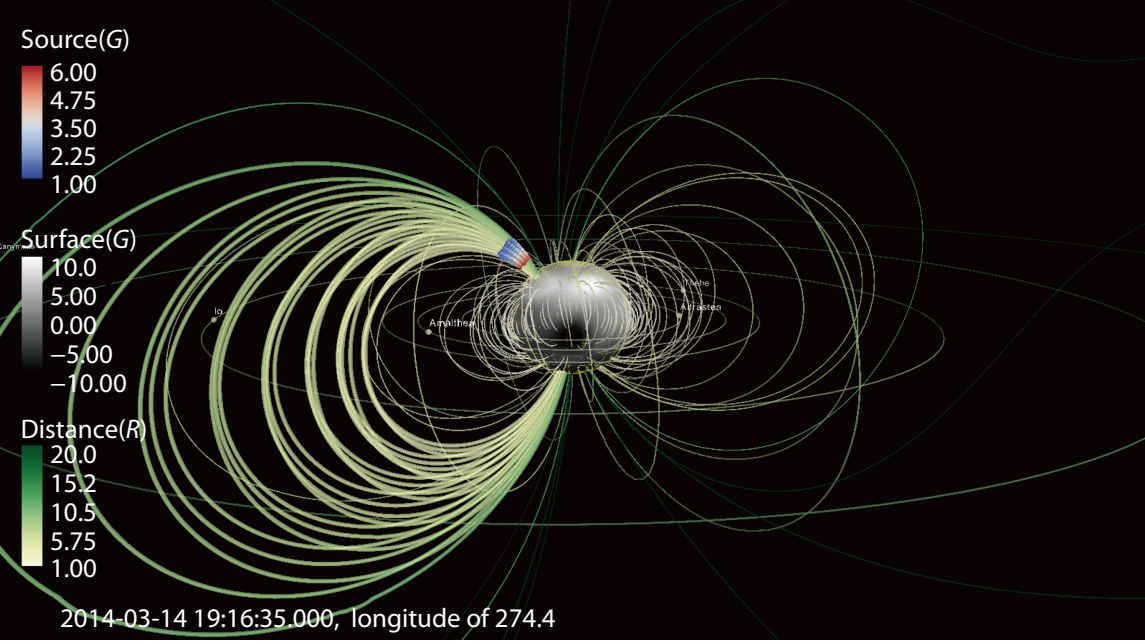

Figure 5. The upper to lower panels show the located DAM source based on the radio dynamic spectrum from Wind/WAVES, ST-A/WAVES and ST-B/WAVES, respectively. The $1 / 15.4$ flattened surface of $B_{r}$ at one $R_{\mathrm{J}}$ is indicated by the gray-scaled ellipsoid at the center and is scaled by the gray bar on the left. Thin curves show the background magnetic field lines with colors, scaled by the green bar on the left, denoting the distances of the tops of these field lines away from the center of Jupiter. All the selected field lines are thicker ones, and the located DAM source corresponding to the emission frequency from $5-16 \mathrm{MHz}$ is marked by color-coded dots along these field lines. The color of the DAM source indicates the local magnetic field strength; it is scaled by the color bar on the left. The major moons, including lo, Europa, Ganymede, Thebe, Amalthea, Adrastea and Metis, as well as their orbits, are marked by small balls and nearly round curves. The time and longitude indicated at the lower-left corner of each panel give the time at Jupiter and the longitude of the disk center of Jupiter, respectively. These images are produced by using Python with Mayavi (Ramachandran and Varoquaux, 2011). An animation can be found in the supplement. 

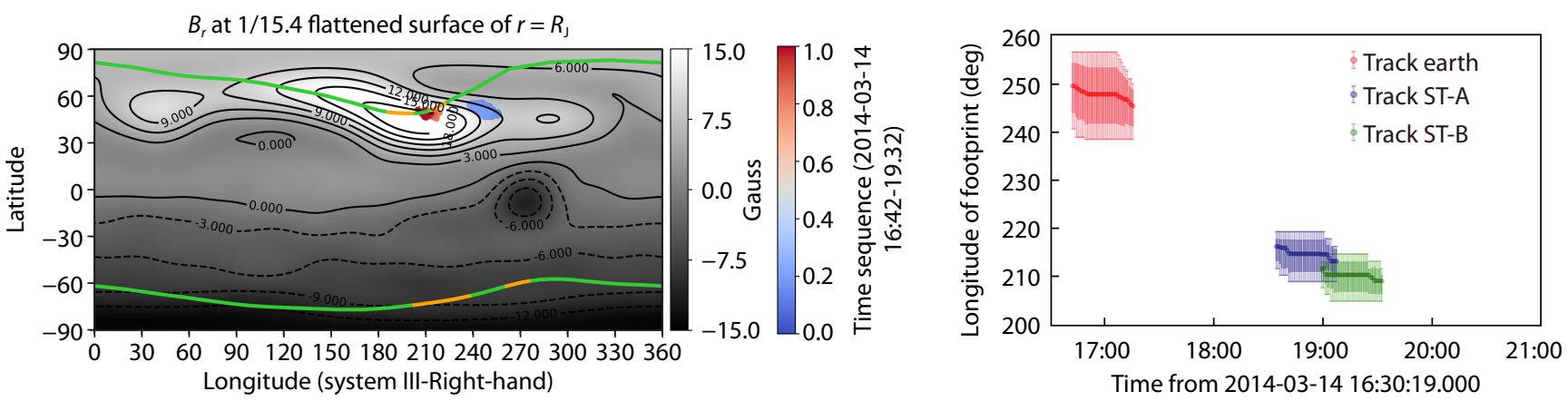

Figure 6. The left panel shows contours of $B_{r}$ in Gauss at the $1 / 15.4$ flattened surface of one $R_{\mathrm{J}}$, which is plotted in the right-handed System III coordinates. The color-coded symbols denote the footprints of selected field lines where the DAM were emitted. The blue-to-red symbols correspond to the earlier to later times as indicated by the color-bar on the right. Concretely, the blue symbols are obtained based on Wind/WAVES observations; the orange and red symbols based on ST-A and ST-B observations, respectively. The two green lines on the map denote the footprints of lo according to the work by Bonfond et al. (2009), and the orange segments mark the footprints of lo when the DAM observed by Wind, ST-A and ST-B. The right panel shows the longitudes of the footprints of these selected field lines. The circles give the median values, the thick bars give the top and bottom 10th percentiles, and the thin bars the minimum and maximum values.
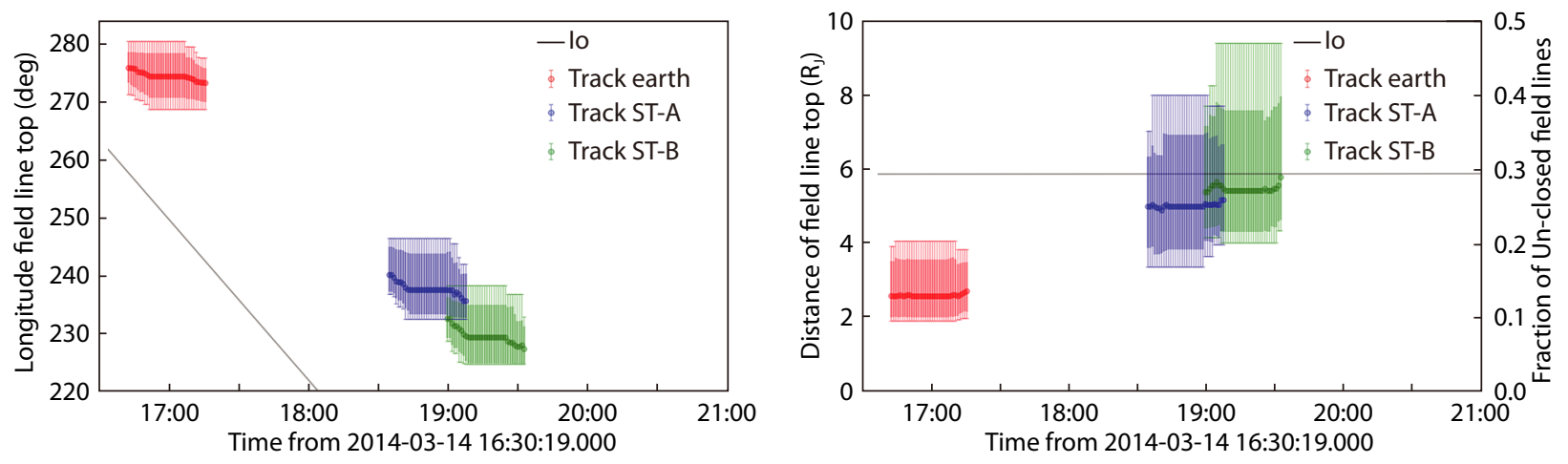

Figure 7. The left panel shows the longitudes of the tops of these selected field lines. For comparison, the gray line indicates the longitude of lo. The right panel shows the distances of the tops of the selected field lines. In our procedure to calculate these values, all the open field lines or field lines with their tops beyond $50 R_{\mathrm{J}}$ are ignored. The fraction of the ignored field lines is indicated by the line scaled by the vertical axis on the right. In this case, the fraction is zero. The circles and bars in both panels have the same meaning as those in the right panel of Figure 6 .
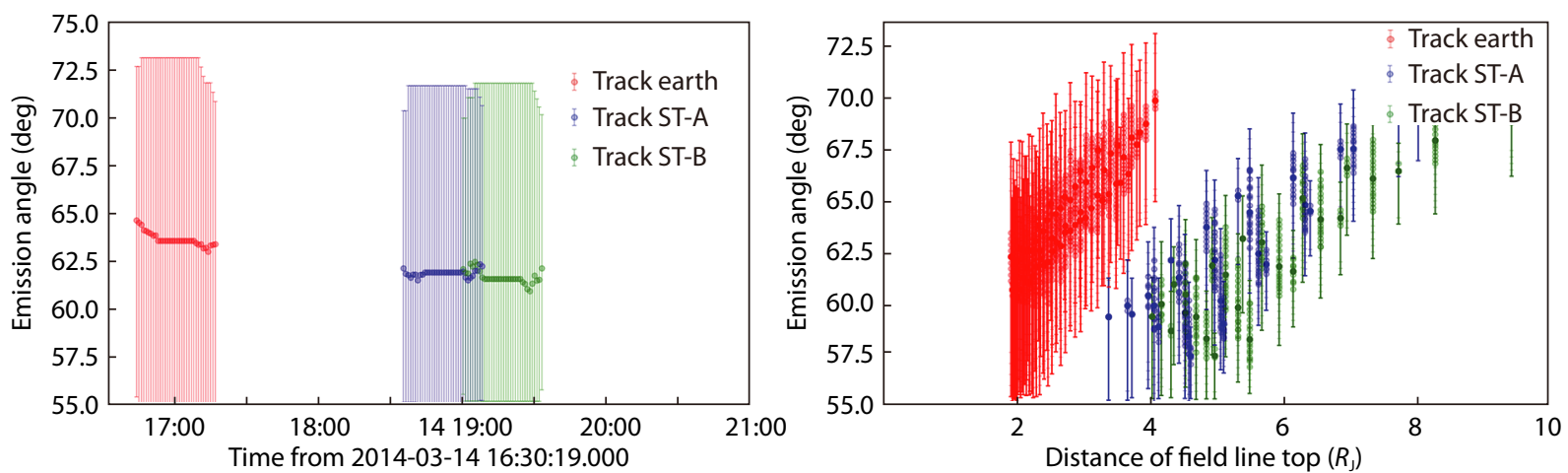

Figure 8. The left panel shows estimated emission cone angles during the DAM emissions. The circles give the median values, and the bars give the minimum and maximum values. The right panel shows the dependence of estimated emission cone angles on the distances of the field line tops. Each circle with an error bar gives the median, minimum and maximum values on a certain field line at a certain time.

different from those of footprints because of the non-pure dipole field of Jupiter and the presence of the tilt angle between the magnetic axis and rotational axis as mentioned before. These longitudes are compared to the longitude of lo to reveal possible relationship between the source field lines and lo. When
Wind/WAVES received the emissions, the longitude was about $24^{\circ}$ larger than that of lo. And 137 minutes later, i.e., when ST$B /$ WAVES received the emission, the difference increased to $42^{\circ}$, suggesting that the apparent rotation speed of the source field line tops, $\Omega_{\text {top }}$, was about $0.45 \Omega$ J, smaller than that of footprints 
but still larger than $\Omega_{c c}$. It should be noted that the apparent rotation speed is not the rotation speed of any given field lines, because the radio source originating from lo activities is not fixed on certain field lines. Moreover, $\Omega_{\text {top }}$ is not an apparent speed at a certain distance, because the source field lines gradually shifted outward as shown in the right panel of Figure 7. The median value of the distances of the tops was about $2.5 R_{\mathrm{J}}$ with the minimum of about $2 R_{\mathrm{J}}$ and maximum of $4 R_{\mathrm{J}}$ when Wind/WAVES received the emission. About two hours later, the source field lines spread over a wider distance range from $3 R_{\mathrm{J}}$ to nearly $10 R_{\mathrm{J}}$ with most source field lines within the distance of $8 R \mathrm{~J}$.

The emission cone angles derived by using Equation (2) through the fitting procedure in our method are displayed in Figure 8. A pattern is clearly revealed in the right panel that the emission angle tends to be smaller on the inner magnetic field lines than that on the outer magnetic field lines. The derived emission angles fall well within the preset range of $50^{\circ}-90^{\circ}$ in the method. Besides, as mentioned before, the averaged emission angle decreased with time during the event as shown in the left panel. This is a cause of that the apparent rotation speed of the source, i.e., $\Omega_{c c}$, was slower than that of the source field lines.

The energy (or the speed) of energetic electrons exciting the DAM emissions is the fitting parameter of Equation (2) and plotted in Figure 9. The preset electron energy is $0.2 \mathrm{keV}$, corresponding to the speed of $0.05 c$. The derived electron energy and speed are well above the limits, suggesting a successful fitting. According to the left panel, it is suggested that when the emission cone swept through Wind spacecraft, the energetic electrons confined in the source field lines had an energy in the range of about 7.5-15.5 $\mathrm{keV}$, and 137 minutes later, when it swept through ST-B, the energy of the associated electrons spread into a slightly wider range of about 9-24 keV. The electron energy falls in the similar range fitted by Hess et al. $(2007,2010)$ using the lo-DAM arcs, but is slightly larger than the energy of electrons emitting the millisecond bursts. The dependence of the electron energy on the distance of the field line top given in the right panel shows the change more clearly. The electrons emitting the DAM observed by ST-A and ST-B were apparently more energized than those observed by Wind, with energy increasing from below $\sim 15 \mathrm{keV}$ to about $24 \mathrm{keV}$. The electrons on the outer magnetic field lines typically had a lower energy than those on the inner magnetic field lines. Moreover, according to Equation (2), the emission angle decreases with the increase of electron energy. Thus, the emission angle on the inner magnetic filed lines was smaller than that on the outer magnetic field lines as shown in the right panel of Figure 8.

\section{Summary and Discussion}

In this paper, we present a method to locate the source of DAM emissions from the Jovian magnetosphere following the work by Hess et al. (2008, 2010). The method only uses the time and frequency of the DAM drift pattern in the radio dynamic spectrum recorded by a spacecraft, and allows us to derive the information of the source location, source field lines, emission cone angle and the energy of associated electrons. If there are multiple spacecraft receiving the DAM emission from different perspectives, the evolution of the DAM source can be revealed.

By applying this method to an lo-DAM observed on 2014 March 14 , we locate the source field lines rooting in a relatively small region in Jupiter's northern ionosphere close to where lo is magnetically mapped, just as expected. Considering the change of emission angles, we find that the rotation speed of the emission source, i.e., the rotation speed of the footprints of identified source field lines, matches that estimated from 2D correlation analysis. The derived emission cone angles and electron energies are well within the preset ranges.

Further integrating the results presented in Section 4, we may outline the following picture for this lo-DAM event. Volcanic activities on lo released neutral particles into space, which then rapidly got ionized, becoming plasma. Through additional acceleration and transport processes and their interaction with Jupiter's magnetosphere, some of the plasma may be energized, producing energetic electrons. These electrons were trapped by magnetic field lines connecting to lo, moved down toward the surface of Jupiter and excited DAM emissions through CM instability. Some electrons were precipitated into the ionosphere, and some were bounced in the field lines and gradually diffused depending on their energies and pitch angles. During this process, the electrons were somehow further energized and spread over a wider region. The diffusive motion of these electrons led to that the DAM source was not fixed on initial field lines, but propagated
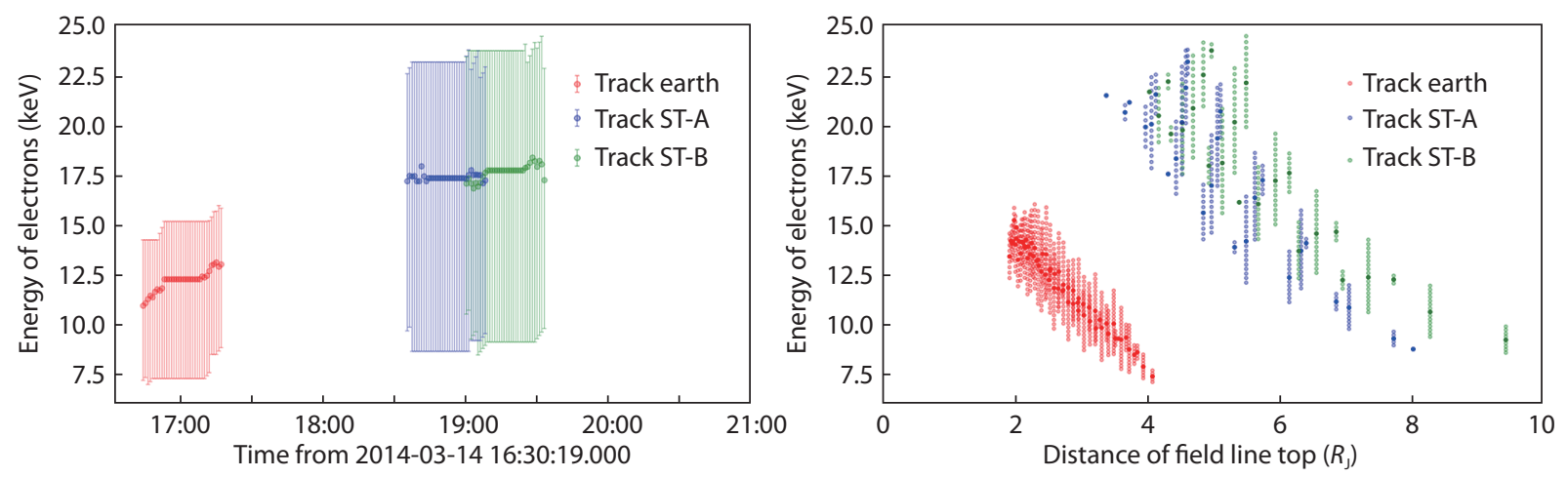

Figure 9. The left panel shows the estimated electron energy. The circles with error bars indicate the median, minimum and maximum values. The right panel shows the dependence of the electron energy on the distances of the field line tops. 
Table 1. Some characteristic parameters.

\begin{tabular}{ccccccccc}
\hline$\Omega_{\mathrm{CC}}$ & $\Omega_{\mathrm{lo}}$ & $\Omega_{\mathrm{IFP}}$ & $\mathrm{LA}^{\mathrm{a}}$ & $\Omega_{\mathrm{fp}}$ & $\Omega_{\mathrm{top}}$ & Distance $/ R_{\mathrm{J}}$ & $\mathrm{EA}^{\mathrm{b}} /\left(^{\circ}\right)$ & Energy/keV $^{\circ}$ \\
\hline $0.255 \Omega_{\mathrm{J}}$ & $0.23 \Omega_{\mathrm{J}}$ & $0.55 \Omega_{\mathrm{J}}$ & $32^{\circ}$ & $0.55 \Omega_{\mathrm{J}}$ & $0.45 \Omega_{\mathrm{J}}$ & $\sim\left(2.5_{-0.5}^{+1.5}-5.5_{-1.5}^{+2}\right)$ & $\sim\left(63.5_{-8.5}^{+9.5}-61.5_{-6.5}^{+10}\right)$ & $\sim\left(12.5_{-5}^{+3}-18_{-9}^{+6}\right)$ \\
\hline
\end{tabular}

a Lead angle of the footprints of the field lines.

b Emission cone angle.

with a pace very close to lo's footprint. The apparent speeds of the footprints and tops of the DAM source field lines were about $0.55 \Omega \mathrm{J}$ and $0.45 \Omega \mathrm{J}$, respectively. The obtained footprint speed is almost the same as that of the IFP on the northern hemisphere with a nearly constant lead angle of about $32^{\circ}$, suggesting a strong connection with lo. All the speeds were larger than the apparent rotation speed of the DAM source derived from 2D crosscorrelation analysis, which was about $0.255 \Omega$, mainly due to the decrease of the emission cone angle. All characteristic parameters obtained in this study are summarized in Table 1. This picture is consistent with our current understanding of lo-DAM emissions (e.g., Zarka, 1998; Hess et al., 2008). All of the above results suggest that our method is valid.

Moreover, it is found that the electrons were more energized when the footprints of the DAM source field lines were located in a stronger field region as can be seen from Figure 6 and 9 . Hess et al. (2010) found a dependence of the electron energy on lo's longitude. We consider this may be due to the variation of the magnetic field intensity of the flux tubes connecting with lo. The larger the magnetic field intensity is, the stronger can be the interaction between lo and Jupiter, and the electrons would likely to be more energized. However, this may also be a result from the assumption used in the model. In Equation (2), the emission angle is a function of the combination of the electron velocity and the magnetic field strength at the top of ionosphere. For this event, the change of the average value of the derived emission angle was small. One can therefore expect that the electron energy derived from Wind observation will be lower than that derived from ST-B observation, since the DAM received by Wind came from a source region with a weaker magnetic field than that by ST-B. Further analysis based on the infrared and UV observations may be helpful to clarify this.

According to the Alfvén wing model of lo-Jupiter magnetic field interaction (Hill et al., 1983), the source field lines not corotating with Jupiter but moving slightly faster than lo is attributed to the presence of lo's ionosphere, of which the electrical conductance, $\Sigma_{l}$, including the Pedersen and Hall conductance, is significant and can slow down Jupiter's field lines passing through it. Previous studies suggested that the conductance is about two orders higher than the Alfvén conductance, $\Sigma_{A}$, outside lo's ionosphere (e.g., Saur et al., 1999; Kivelson et al., 2004). For such a high conductance, the field lines in the vicinity of lo would be dragged to almost corotate together with lo for a while. When the field lines are dragged by lo, Alfvén wings will form and result in the longitude difference between the source field lines and lo, which is called Mach angle and is typically less than $10^{\circ}$ (refer to Fig. 10.3 in Hill et al. (1983)). For this event, our method shows that the tops of source field lines located ahead of lo with a lead angle from about $24^{\circ}$ at Wind to about $42^{\circ}$ at ST-B. This is different from that for the footprints of the source field lines, of which the lead angle is about $32^{\circ}$ without significant change during the event.

Besides, at the earlier time, i.e., when the DAM emission lighted Wind spacecraft, the source field lines were within the lo orbit. We are not sure if this is true or resulted from some uncertainties in our method. If it is true, the energetic electrons should be injected and/or quickly transported into inner magnetic field lines through some process. A possible scenario is as follows. lo ejected plasmas into magnetosphere. These plasmas were trapped by and corotated with Jupiter's field lines, and stretched these field lines outward due to the centrifugal force. The stretched field lines began to reconnect and convect inward at a certain time and consequently generated energetic electrons. These electrons were further transported into the inner magnetosphere within the lo orbit through cross-field diffusion. However, since only the static magnetospheric field model is used in our method, we are unable to distinguish any detailed dynamic processes.

\section{Acknowledgments}

We acknowledge the use of the data from the radio instruments on board Wind, STEREO-A and B spacecraft. We thank the anonymous referees for many valuable comments. We are also grateful to Hao Cao from Harvard University for valuable discussions. The work is support by the Strategic Priority Program of the Chinese Academy of Sciences (Grant Nos. XDB41000000, XDA15017300) and the NSFC (Grant No.41842037 and 41574167). V. Krupar acknowledges support by an appointment to the NASA postdoctoral program at the NASA Goddard Space Flight Center administered by Universities Space Research Association under contract with NASA and the Czech Science Foundation grant 1706818Y.

\section{References}

Bagenal, F. (1994). Empirical model of the lo plasma torus: Voyager measurements. J. Geophys. Res.: Space Phys., 99(A6), 11043-11062. https://doi.org/10.1029/93JA02908

Bolton, S. J., Lunine, J., Stevenson, D., Connerney, J. E. P., Levin, S., Owen, T. C., Bagenal, F., Gautier, D., Ingersoll, A. P., ... Thorpe, R. (2017). The Juno mission. Space Sci. Rev., 213(1-4), 5-37. https://doi.org/10.1007/s11214-0170429-6

Bonfond, B., Grodent, D., Gérard, J. C., Radioti, A., Saur, J., and Jacobsen, S. (2008). UV lo footprint leading spot: A key feature for understanding the UV lo footprint multiplicity?. Geophys. Res. Lett., 35(5), L05107. https://doi.org/10.1029/2007GL032418

Bonfond, B., Grodent, D., Gérard, J. C., Radioti, A., Dols, V., Delamere, P. A., and Clarke, J. T. (2009). The lo UV footprint: Location, inter-spot distances and tail vertical extent. J. Geophys. Res.: Space Phys., 114(A7), A07224. https://doi.org/10.1029/2009JA014312

Bougeret, J. L., Kaiser, M. L., Kellogg, P. J., Manning, R., Goetz, K., Monson, S. J., Monge, N., Friel, L., Meetre, C. A., ... Hoang, S. S. (1995). WAVEs: The radio and plasma wave investigation on the wind spacecraft. Space Sci. Rev., 71(1- 


\section{4), 231-263. https://doi.org/10.1007/BF00751331}

Bougeret, J. L., Goetz, K., Kaiser, M. L., Bale, S. D., Kellogg, P. J., Maksimovic, M., Monge, N., Monson, S. J., Astier, P. L., ... Zouganelis, I. (2008). S/WAVES: The radio and plasma wave investigation on the STEREO mission. Space Sci. Rev., 136(1-4), 487-528. https://doi.org/10.1007/s11214-007-9298-8

Carr, T. D., Desch, M. D., and Alexander, J. K. (1983). Phenomenology of magnetospheric radio emissions. In A. J. Dessler (Ed.), Physics of Jovian Magnetosphere (pp. 226-284). New York: Cambridge University Press.

Connerney, J. E. P. (1992). Doing more with Jupiter's magnetic field. In H. O. Rucker, et al. (Eds.), Planetary Radio Emissions III (pp. 13-33). Vienna: Austrian Academy of Science.

Connerney, J. E. P., Acuña, M. H., and Ness, N. F. (1981). Modeling the Jovian current sheet and inner magnetosphere. J. Geophys. Res.: Space Phys., 86(A10), 8370-8384. https://doi.org/10.1029/JA086iA10p08370

Connerney, J. E. P., Kotsiaros, S., Oliversen, R. J., Espley, J. R., Joergensen, J. L., Joergensen, P. S., Merayo, J. M. G., Herceg, M., Bloxham, J., ... Levin, S. M. (2018). A new model of Jupiter's magnetic field from Juno's first nine orbits. Geophys. Res. Lett., 45(6), 2590-2596. https://doi.org/10.1002/2018GL077312

Cowley, S. W. H., and Bunce, E. J. (2001). Origin of the main auroral oval in Jupiter's coupled magnetosphere-ionosphere system. Planet. Space Sci., 49(10-11), 1067-1088. https://doi.org/10.1016/S0032-0633(00)00167-7

Dulk, G. A., Leblanc, Y., and Lecacheux, A. (1994). The complete polarization state of lo-related radio storms from Jupiter: A statistical study. Astron. Astrophys., 286, 683-700.

Giampieri, G., and Dougherty, M. K. (2004). Modelling of the ring current in Saturn's magnetosphere. Ann. Geophys., 22(2), 653-659. https://doi.org/10.5194/angeo-22-653-2004

Grodent, D., Bonfond, B., Gérard, J. C., Radioti, A., Gustin, J., Clarke, J. T., Nichols, J., and Connerney, J. E. P. (2008). Auroral evidence of a localized magnetic anomaly in Jupiter's northern hemisphere. J. Geophys. Res.: Space Phys., 113(A9), A09201. https://doi.org/10.1029/2008JA013185

Hess, S., Zarka, P., and Mottez, F. (2007). lo-Jupiter interaction, millisecond bursts and field-aligned potentials. Planet. Space Sci., 55(1-2), 89-99. https://doi.org/10.1016/j.pss.2006.05.016

Hess, S., Cecconi, B., and Zarka, P. (2008). Modeling of lo-Jupiter decameter arcs, emission beaming and energy source. Geophys. Res. Lett., 35(13), L13107. https://doi.org/10.1029/2008GL033656

Hess, S. L. G., Pétin, A., Zarka, P., Bonfond, B., and Cecconi, B. (2010). Lead angles and emitting electron energies of lo-controlled decameter radio arcs. Planet. Space Sci., 58(10), 1188-1198. https://doi.org/10.1016/j.pss.2010.04.011

Hess, S. L. G., Echer, E., Zarka, P., Lamy, L., and Delamere, P. A. (2014). Multiinstrument study of the Jovian radio emissions triggered by solar wind shocks and inferred magnetospheric subcorotation rates. Planet. Space Sci., 99, 136-148. https://doi.org/10.1016/j.pss.2014.05.015

Hill, T. W., Dessler, A. J., and Goertz, C. K. (1983). Magnetospheric models. In A. J. Dessler (Ed.), Physics of the Jovian Magnetosphere (pp. 353-394). Cambridge: Cambridge University Press.

Imai, K., Wang, L. Y., and Can, T. D. (1997). Modeling Jupiter's decametric modulation lanes. J. Geophys. Res.: Space Phys., 102(A4), 7127-7136. https://doi.org/10.1029/96JA03960

Imai, K., Riihimaa, J. J., Reyes, F., and Carr, T. D. (2002). Measurement of Jupiter's decametric radio source parameters by the modulation lane method. J. Geophys. Res.: Space Phys., 107(A6), SMP 12-1-SMP 12-11. https://doi.org/10.1029/2001JA007555

Jacobsen, S., Neubauer, F. M., Saur, J., and Schilling, N. (2007). Io's nonlinear MHD-wave field in the heterogeneous Jovian magnetosphere. Geophys. Res. Lett., 34(10), L10202. https://doi.org/10.1029/2006GL029187

Kaiser, M. L., Zarka, P., Kurth, W. S., Hospodarsky, G. B., and Gurnett, D. A. (2000).
Cassini and Wind stereoscopic observations of Jovian nonthermal radio emissions: Measurement of beam widths. J. Geophys. Res.: Space Phys., 105(A7), 16053-16062. https://doi.org/10.1029/1999JA000414

Kivelson, M. G., and Southwood, D. J. (2005). Dynamical consequences of two modes of centrifugal instability in Jupiter's outer magnetosphere. J. Geophys. Res.: Space Phys., 110(A12), A12209. https://doi.org/10.1029/2005JA011176

Kivelson, M. G., Bagenal, F., Kurth, W. S., Neubauer, F. M., Paranicas, C., and Saur, J. (2004). Magnetospheric interactions with satellites. In F. Bagenal et al. (Ed.), Jupiter: The Planet, Satellites and Magnetosphere (pp. 513-536). Cambridge: Cambridge University Press.

Lamy, L., Zarka, P., Cecconi, B., Hess, S., and Prangé, R. (2008). Modeling of Saturn kilometric radiation arcs and equatorial shadow zone. J. Geophys. Res.: Space Phys., 113(A10), A10213. https://doi.org/10.1029/2008JA013464

Lamy, L., Prangé, R., Pryor, W., Gustin, J., Badman, S. V., Melin, H., Stallard, T., Mitchell, D. G., and Brandt, P. C. (2013). Multispectral simultaneous diagnosis of Saturn's aurorae throughout a planetary rotation. J. Geophys. Res.: Space Phys., 118(8), 4817-4843. https://doi.org/10.1002/jgra.50404

Lecacheux, A. (1988). Polarization aspects from planetary radio emissions. $\ln \mathrm{H}$. O. Rucker, et al. (Eds.), Planetary Radio Emissions II (pp. 311-326). Vienna: Austrian Academy of Science.

Panchenko, M., and Rucker, H. O. (2016). Estimation of emission cone wall thickness of Jupiter's decametric radio emission using stereoscopic STEREO/WAVES observations. Astron. Astrophys., 596, A18. https://doi.org/10.1051/0004-6361/201527397

Queinnec, J., and Zarka, P. (1998). lo-controlled decameter arcs and lo-Jupiter interaction. J. Geophys. Res.: Space Phys., 103(A11), 26649-26666. https://doi.org/10.1029/98JA02435

Ramachandran, P., and Varoquaux, G. (2011). Mayavi: 3D visualization of scientific data. Comput. Sci. Eng., 13(2), 40-51. https://doi.org/10.1109/MCSE.2011.35

Riihimaa, J. J. (1968). Structured events in the dynamic spectra of Jupiter's decametric radio emission. Astron. J., 73, 265-270. https://doi.org/10.1086/110627

Riihimaa, J. J. (1978). L-bursts in Jupiter's decametric radio spectra. Astrophys. Space Sci., 56(2), 503-518. https://doi.org/10.1007/BF01879581

Saur, J., Neubauer, F. M., Strobel, D. F., and Summers, M. E. (1999). Threedimensional plasma simulation of lo's interaction with the lo plasma torus: Asymmetric plasma flow. J. Geophys. Res.: Space Phys., 104(A11), 25105-25126. https://doi.org/10.1029/1999JA900304

Schneider, N. M., and Bagenal, F. (2007). lo's neutral clouds, plasma torus, magnetospheric interactions. In R. M. C. Lopes, et al. (Eds.), lo after Galileo (pp. 265-286). Berlin, Heidelberg: Springer. https://doi.org/10.1007/978-3540-48841-5_11

Treumann, R. A. (2006). The electron-cyclotron maser for astrophysical application. Astron. Astrophys. Rev., 13(4), 229-315. https://doi.org/10.1007/s00159-006-0001-y

Waite, J. H. Jr., Clarke, J. T., Cravens, T. E., and Hammond, C. M. (1988). The Jovian aurora: Electron or ion precipitation?. J. Geophys. Res.: Space Phys., 93(A7), 7244-7250. https://doi.org/10.1029/JA093iA07p07244

Wu, C. S., and Lee, L. C. (1979). A theory of terrestrial kilometric radiation. Astrophys. J., 230, 621-626. https://doi.org/10.1086/157120

Zarka, P. (1998). Auroral radio emissions at the outer planets: Observations and theories. J. Geophys. Res.: Plants, 103(E9), 20159-20194. https://doi.org/10.1029/98JE01323

Zarka, P., Farges, T., Ryabov, B. P., Abada-Simon, M., and Denis, L. (1996). A scenario for Jovian S-bursts. Geophys. Res. Lett., 23(2), 125-128. https://doi.org/10.1029/95GL03780 